\title{
A Study on the Irregularity of the Sequence of R-R Intervals in Chronic Atrial Fibrillation in Man Based on the Time Series Analysis and the Information Theory
}

\author{
Etsu Hashida, M.D., Naohiro Yoshitani, Ph.D., and \\ Takenobu Tasakr, Ph.D.
}

\section{Summary}

The mechanism of the irregularity of R-R intervals and especially the genesis of the long $R-R$ intervals in atrial fibrillation were clarified. From the point of view of the present study, the sequence of successive $R$ $\mathrm{R}$ intervals can be regarded as a stochastic process, and consequently mathematical analyses can be applied, and the following conclusion could be obtained : the stability of the data, mathematically called stationarity, is present in the sequence of $R-R$ intervals, and these intervals are independently and identically distributed. The probability density function is an Erlangian distribution with phase $\mathrm{k}$ of 20 to 26 . Namely, as soon as, on the average, 20 to 26 atrial excitatory stimuli have arrived at the $\mathrm{AV}$ node, a threshold value of the AV node is reached and ventricular activation is induced. However, if we examine the mathematical structure of the sequence of successive $R-R$ intervals in more detail, the following results can be evidently obtained: during atrial fibrillation with rapid ventricular response, the sequence of $R-R$ intervals is completely independent, but with intermediate or slow ventricular response the adjacent $\mathrm{R}-\mathrm{R}$ intervals correclate with each other and these adjacent intervals have at least the first order Markov property, which seems to be caused by the concealed conduction. Because of this Markov property due to the prolongation of the refractory period of the AV node, slow ventricular response results.

\section{Aditional Indexing Words :}

Arrhythmia Atrial fibrillation Artioventricular node Concealed conduction

From the Department of Internal Medicine, Health Insurance Hospital of Shiga, Ohtsu, Japan 520 and the Computing Center, Shionogi Research Laboratory, Osaka, Japan 553.

This paper was presented in part at the Annual Meeting of the Japanese Circulation Society, Yamaguchi, April, 1974.

Address for reprint: Etsu Hashida, M.D., Health Insurance Hospital of Shiga, Fujimidai, Ohtsu, Japan 520.

Received for publication May 8, 1978.

Revision accepted July 11, 1978. 
7 HE mechanism of the irregular ventricular response and especially the genesis of the long $\mathrm{R}-\mathrm{R}$ intervals during atrial fibrillation are still poorly understood. Although the mathematical structure of the irregularity of the sequence of $\mathrm{R}-\mathrm{R}$ intervals seems to be the most suitable problem to be challenged by such sophisticated methods of analysis as probability theory, ${ }^{1,2)}$ time series analysis, ${ }^{2), 3)}$ and information theory, ${ }^{4)}$ many controversial and obscure points remain unsolved. The previous paper of the authors ${ }^{5)}$ demonstrated as a first step that the sequence of $R-R$ intervals in atrial fibrillation with slow ventricular response was independently and identically distributed, that is, the sequence could be described as a renewal process. In that paper, it was also shown that the serial correlation coefficients of the sequence of R-R intervals were not significantly different from zero and that the spectrum of intervals could be estimated as constant.

The results of both the serial correlation coefficients and the spectrum of intervals indicate that the necessary conditions for the independency of the successive $R-R$ intervals associated with atrial fibrillation are satisfied but the results thus estimated do not always become sufficient conditions for the independency of these intervals. ${ }^{6)-81}$ In the present study, therefore, the necessary and sufficient conditions for the dependency of the sequence of R-R intervals were presented with respect to the mathematical structure of these intervals using information theory in addition to probability theory and time series analysis. The order of Markov property providing a measure of dependency between these intervals, if the dependency was present, was calculated in cases of rapid, intermediate, and slow ventricular response before, during, and after digitalization, respectively.

\section{Materials and Methods}

Three electrocardiographic records, consisting of about 2,000 successive intervals were derived from continuous recording of electrocardiograms of a 73year-old male patient with aortic insufficiency and atrial fibrillation. Before, during, and after digitalization, the electrocardiograms of about $30 \mathrm{~min}$ were recorded at a mean ventricular response of 100,75 , and $56 \mathrm{~min}$, respectively. The time intervals between the summits of $\mathrm{R}$ waves were measured manually (in $\mathrm{msec}$ ). For each electrocardiographic record, interval histograms with bin width $200 \mathrm{msec}$, and serial correlation coefficients and spectral density functions of the sequence of successive R-R intervals were calculated.

A general expression for the expected $R-R$ interval distribution is not known, but under several restrictions the following model can be derived : if the decay of the AV nodal depolarization between excitatory impulses is negligible, if excitatory atrial inputs are exponentially distributed, and if the AV nodal depolarization reaches a threshold of, say, $\mathrm{k}$ units, the distribution can be derived exactly.9" For all $k$, the exact form is a well-known Erlangian distribution. ${ }^{1), 2), 8), 9)}$ First, the 
configurations of R-R interval histograms were approximated by an Erlangian distribution. The value of $\mathrm{k}$, the phase of the distribution, most appropriately fitted to the actual histogram was estimated with inspection and by chi-squared test when $\mathrm{k}$ becomes large. (For the outline of mathematical treatment we refer the readers to the Appendix.) Second, concerning the test of stationarity necessary for data processing including the calculations of serial correlation coefficients, spectral density functions and so on, we refer the readers to our previous paper ${ }^{5)}$ and others. ${ }^{2,3)}$ Yet, for the convenience, these terms are explained briefly: each correlation coefficient is a measure of degree of relationship between adjacent or non-adjacent intervals, and the spectral density function represents the distribution by frequency composition of the data (in this case, $1 / R-R$ interval) in terms of the mean square values of the data (in this case, (R-R interval) ${ }^{2}$ ).

Furthermore, in the present study, we determined the order of Markov process of the sequence of $R-R$ intervals as a measure of dependency between these successive intervals using information theory.

The most fundamental approach is to determine whether the sequence of intervals is independently and identically distributed. When the intervals are drawn independently from a common probability density function, the stochastic process which forms the sequence of these intervals is called a renewal process and the entire process is fully characterized by the probability density function. However, the stationary stochastic process which is usually studied by time series analysis is not always independently distributed, even if the process may be identically distributed. The Markov process is one of such processes. When the interval is influenced only by the immediately preceding interval, the first order Markov property is present, and when the interval correlates with 2 successive intervals which immediately precede the interval in question, the second order Markov property is present, and so on. ${ }^{10}$, (Those who desire fuller discussion of the text are referred to the Appendix in advance.)

The order of Markov process of the sequence is determined by the conditional entropy which is derived from the joint entropy. For the sake of simplicity, the Gaussian process is assumed, and here the joint entropy is directly related with the covariance matrix which can be expressed by the matrix of the serial correlation coefficients of R-R intervals. Therefore, the condition to determine the order of Markov process is obtained by the equation of the matrices of the serial correlation coefficients.

We denote the serial correlation coefficients by $\rho_{1}, \rho_{2}, \ldots, \rho_{\mathrm{n}}$, and call $\rho_{\mathrm{i}}$ the $\mathrm{i}$-th order serial correlation coefficient. Further, we define by $\mathrm{S}^{\mathrm{n}}$ the following matrix consisting of the serial correlation coefficients :

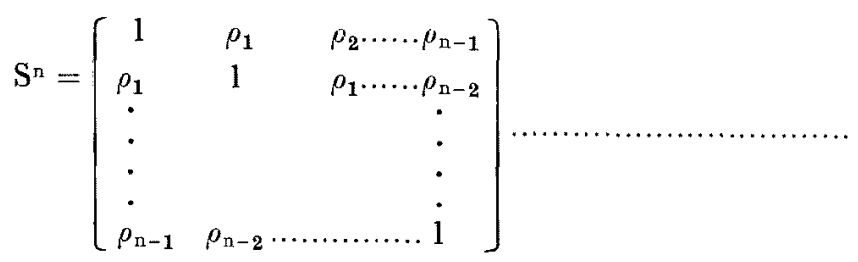

Then, in order to estimate the order of Markov process, it is necessary to test whether the equation of matrices 


$$
\left|\mathbf{S}^{\mathrm{n}-1}\right|-\left|\mathrm{S}^{\mathrm{n}-2}\right|^{1 / 2}\left|\mathrm{~S}^{\mathrm{n}}\right|^{1 / 2}=0
$$

holds for all integers n's. For that purpose, the variability of the value of Eq. (2) is tested by the shuffling procedure. As shown by Perkel et al, ${ }^{11)}$ if the original sequence of R-R intervals is randomly shuffled, the shuffled sequence loses the statistical dependency.

Let $S_{o}^{m}$ be the matrix of the original serial correlation coefficients as defined in (1), and similarly $S_{s}^{m}$, that of the serial correlation coefficients of the shuffled sequence. Here we define $\varepsilon_{\mathrm{m}}$ and $\delta_{\mathrm{m}}$ by

$$
\begin{aligned}
& \varepsilon_{\mathrm{m}}=\left|\mathrm{S}^{\mathrm{m}-1}\right|-\left|\mathrm{S}^{\mathrm{n}-2}\right|^{1 / 2}\left|\mathrm{~S}_{o}^{\mathrm{m}}\right|^{1 / 2}, \\
& \delta_{\mathrm{m}}=\left|\mathrm{S}^{\mathrm{m}-1}\right|-\left|\mathrm{S}^{\mathrm{m}-2}\right|^{1 / 2}\left|\mathrm{~S}_{\mathrm{s}}^{\mathrm{m}}\right|^{1 / 2}
\end{aligned}
$$

If the following inequality

$$
\varepsilon_{\mathrm{ma}} \leqq \delta_{\mathrm{m}}
$$

holds for all integers $m^{\prime} \geqq m$, the sequence is less than or equal to the $(m-1)$-th order Markov process. If the following inequality

$$
\varepsilon_{\mathrm{m}}>\delta_{\mathrm{m}}
$$

holds, the sequence is more than the $(\mathrm{m}-\mathrm{l})$-th order Markov process.

For the computation of the variability of $\delta_{\mathrm{m}}, 30$ shuffled sequences were used in this study. Thus the range of the value of $\delta_{\mathrm{m}}$ was determined with the values of $3 \sigma$ and $-3 \sigma$, where $\sigma$ is the standard deviation of $\delta_{\mathrm{m}}$.

\section{Results}

The serial correlation coefficients and the spectral density functions of the sequence of $R-R$ intervals observed in a patient with atrial fibrillation were not significantly different from zero and could be regarded as constant, respectively, irrespective of the mean ventricular response per minute. That is, the sequence of $R-R$ intervals in atrial fibrillation could be considered as a renewal process and the probability density function explaining the configurations of histograms could be obtained as an Erlangian distribution whose phase $\mathrm{k}$ was most appropriately fitted with $\mathrm{k}$ equal to 20 to 26 irrespective of the mean ventricular response per minute. The results of the analysis for sample sequences of R-R intervals for these 3 cases are shown in Table $I$.

The presence or absence of Markov property in the sequence of $R-R$ intervals was tested by comparing the values of the determinants of matrices consisting of their serial correlation coefficients concerning both the original sequence and the 30 shuffled ones, and the order of Markov process of the sequence of R-R intervals was estimated. In Fig. 1, the open and filled circles show the values of the second and the first term of the left side of Eq. (2), respectively. The value of the second term of the right side of Eq. 
Table I. Analysis of Sample Sequences of R-R Intervals

\begin{tabular}{c|rrrrrrrrr}
\hline $\begin{array}{c}\text { Number } \\
\text { of R-R } \\
\text { Intervals }\end{array}$ & $f$ & $\mu$ & $\sigma$ & $\sigma / \mu$ & $\rho_{1}$ & $\rho_{2}$ & $\rho_{3}$ & $\rho_{4}$ & $\rho_{5}$ \\
\hline 2,545 & 100 & 604.59 & 14.59 & 0.01896 & 0.044 & 0.041 & 0.003 & 0.019 & 0.009 \\
2,149 & 75 & 794.79 & 162.99 & 0.20507 & 0.132 & 0.096 & 0.058 & 0.054 & 0.062 \\
1,709 & 56 & $1,075.95$ & 231.17 & 0.21485 & 0.079 & -0.006 & 0.026 & -0.005 & 0.016
\end{tabular}

$\mathrm{f}=$ mean frequency of QRS complex, QRS complexes/min, $\mu=$ mean R.R interval, msec, $\sigma=$ standard deviation of (adjacent) R-R intervals, msec, $\sigma / \mu=$ coefficient of variation, $\rho_{1}=$ the first order serial correlation coefficient, $\rho_{2}=$ the second, $\rho_{3}=$ the third, $\rho_{4}=$ the fourth, $\rho_{5}=$ the fifth.

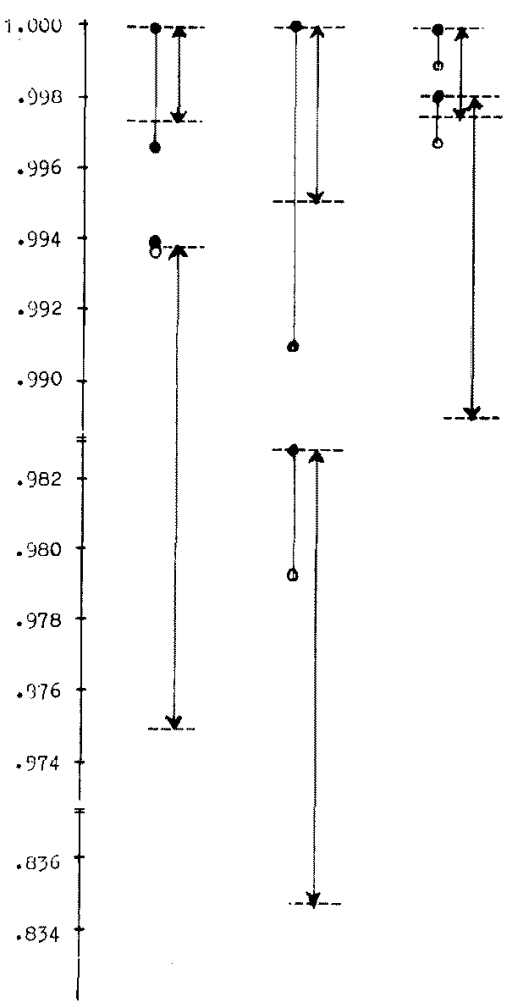

Fig. 1. Estimation of the order of Markov process of the sequence of R-R intervals in atrial fibrillation. The open and filled circles show the value of the second and the first term of the left side of $\mathrm{Eq}$. (1). The value of the second term of the right side of Eq. (3) ranges from the upper broken line to the lower one as shown by the arrowed line. From left to right, the order of Markov process was estimated at the mean ventricular response of 56,75 , and $100 / \mathrm{min}$, respectively. In each column, the first order Markov process was estimated in the upper part and the second one was estimated in the lower part. 
ranges from the upper broken line to the lower one as shown by the arrowed line. Then the Markov property was absent in atrial fibrillation with rapid ventricular response of $100 / \mathrm{min}$, and consequently the sequence of $R-R$ intervals was independent. In cases of slow and intermediate ventricular response of 56 and $75 / \mathrm{min}$, respectively, there existed the first order Markov property, but the second order one did not exist in either case as shown in Fig. 1.

\section{Discussion}

The aims of this paper concerning atrial fibrillation are threefold: (1) to clarify the mathematical structure of the irregularity of the sequence of $\mathrm{R}-\mathrm{R}$ intervals; (2) to analyze the mechanism of slow ventricular response; (3) to estimate the portion in the $\mathrm{AV}$ node upon which digitalis exerts its action. In a certain sense we intended a modeling of the AV node.

Although in atrial fibrillation the mode of generation of impulses in the atria and that of the transmission to the AV node and the propagation through this node of these impulses are not clear, an assumption may be possible that miniature excitations randomly generated at many sites in the fibrillating atria are transmitted to the AV node via a number of pathways. If this assumption is accepted, the distribution of arrival time intervals of these miniature stimuli to the AV node through each pathway may be regarded, as a whole, as exponential as a consequence of superposition of several similar stochastic sequences of simpler forms as viewed from the side of the AV node. ${ }^{1,2), 5)}$

Using terms of the theory of queueing, ${ }^{12)}$ the atrial inputs are positive pulses, and possess such properties as stationarity, absence of after-effects and orderliness. Consequently, the distribution of arrival time intervals of these miniature stimuli is exponential, the potential level of the AV node jumps by one unit at the time of arrival of a miniature input pulse and retains its value until the next arrival. If a negative atrial input is assumed to be absent, 20 to 26 positive inputs (pulses) which arrive at the AV node and are stored are thought to be necessary for this threshold model to fire, that is, for the AV node to elicit ventricular activation. Such problems belong to the well-known first passage time problems in probability theory. ${ }^{8)}$ Their solutions can be obtained by solving the random walk model as explained in the above discussion and further the diffusion equation as its limit. ${ }^{8), 13)-15}$ )

In atrial fibrillation with rapid ventricular response $(100 / \mathrm{min})$ before digitalization, the sequence of successive $R-R$ intervals is independent (zeroth order Markov property). With decreasing ventricular response (56 to 
$75 / \mathrm{min}$ ) during and after digitalization, it is made clear by the present mathematical analysis that the sequence of successive $R-R$ intervals has at least the first order Markov property. These results can be interpreted to correspond to the concealed conduction in the AV node. ${ }^{16)-18}$ ) Furthermore, the presence of Markov property can be thought to be not due to slow ventricular response, but the latter in atrial fibrillation seems to be the result from the Markov property of the sequence of successive R-R intervals (that is to say, the AV node preserves the past memory or the physiological state of the AV node depends highly on its past activity, and the preceding $R-R$ interval exerts influence upon the present interval).

As an example of the above considerations, the mechanism of the irregular R-R intervals associated with atrial fibrillation in isolated rabbit heart was demonstrated by Moore ${ }^{16)}$ to be the concealed conduction of atrial stimuli in the $A V$ node by recording electrograms from right atrium and ventricle simultaneously with transmembrane action potentials from single fibers within the AV node and right bundle branch. The most frequently recorded $R-R$ interval during atrial fibrillation was found by him when a single response was concealed within the AV node. In several experiments of Moore, as many as 7 to 9 consecutively concealed responses were recorded from the AV node (see Figs. 3-5 and Fig. 7 of Moore's paper).

Stein ${ }^{19)}$ notes the following examples of effects which will prevent the sequence of firings from being described by a renewal model. The first example is the accumulation of refractoriness from one interspike interval to the next. As shown in Figs. 3-5 of the paper of Moore, at least 1 response is concealed between 2 consecutive action potentials which occur according to an all-or-none law in the NH region. This concealment can be presumed to correspond to the accumulation of refractoriness and so the distribution of the successive R-R intervals during atrial fibrillation can not necessarily be described as a renewal model. Further, a second example of Stein is a case of the arrival of inputs that extend over more than one interval. This seocnd kind of effect is encountered in a selective-interaction model of the synaptic transmission of impulses described by Ten Hoopen and Reuver. ${ }^{20}$ According to the analysis of Bishop et al ${ }^{21}$ on the properties of the spontaneous firing activity of single neurons in the lateral geniculate nucleus of adult anesthetized cats during darkness observed by Levick and Williams, ${ }^{22}$ photic stimuli are integrated at the ganglion cells in the retina and these integrated output signals are transmitted to the lateral geniculate nucleus where a counteraction with inhibitory inputs occurs. If we take into consideration the above cited paper of Moore, excitatory stimuli from the fibrillating atria could be assumed to be integrated within the $\mathrm{N}$ region of the $\mathrm{AV}$ node, to 
counteract with inhibitory stimuli brought about by the action of digitalis at the boundary between the $\mathrm{N}$ and $\mathrm{NH}$ regions of the $\mathrm{AV}$ node. Consequently, transmembrane action potentials recorded from the $\mathrm{NH}$ region could occur according to an all-or-none law. If the above considerations are valid, slow ventricular response in atrial fibrillation could be reasonably explained by this complex mechanism. Of course, in this instance, the distribution of the sequence of $\mathrm{R}-\mathrm{R}$ interals is not a renewal process and the Markov property between successive intervals could be expected to result.

Also, the first kind of effect noted by Stein might be more appropriately thought as the accumulation of hyperpolarization. With respect to the AV conduction, the effect concerned corresponds to the experimental result suggested by Mendez and Moe ${ }^{23}$ ) that during critical propagation of excitaiton the impulse may stop and resume its travel after a delay but, when propagation starts again, the newly excited tissue will provide depolarizing current to the previously active region, causing a delay in repolarization. In atrial fibrillation it is assumed that such a mechanism as a delay in repolarization or an accumulation of hyperpolarization plays an important role and therefore the dependency between successive $R-R$ intervals is caused.

When superposition of another independent inhibitory inputs upon the original excitatory ones could be assumed, then the solution of the corresponding random walk model might be obtained. ${ }^{24}$ ) Under the assumption that a parameter of the inhibitory inputs arriving at the AV node according to a Poisson distribution is proportional to the level of the transmembrane potential of the AV node, the main results are as follows ${ }^{24)}$ : if the intervals between inputs are exponentially distributed: (1) when inputs reach the AV node at low rates, the intervals between outputs arc also exponential; (2) when inputs reach the AV node at high rates, the intervals between outputs become Gamma-distributed, and the higher the rate of inputs, the greater the order of Gamma distribution regulating the output signals becomes.

Because the action of digitalis is to depress the conductivity and excitability of the AV node, the assumption that inhibitory inputs to the AV node are generated by the administration of this drug may be accepted.

If the atrial stimuli transmitted to the AV node during atrial fibrillation are exponentially distributed and if the intervals between outputs, that is, $\mathrm{R}-\mathrm{R}$ intervals occurring after the arrival of a certain number of these stimuli can be considered as Erlangian-distributed as shown in the present study, the solution of random walk model under appropriate conditions is expected to give more realistic and quantitative estimates of the function of the AV node.

It was previously noted that we used the simplest threshold model of the 
AV node which allowed for the temporal summation of only excitatory stimuli up to a threshold value such that when the amount of excitation stored in the AV node reaches a certain threshold, the AV node transmits the excitatory impulse sufficient to elicit ventricular activation. And also evidence suggesting that summation is a feature of $\mathrm{AV}$ nodal conduction was presented by Zipes et al. ${ }^{251}$

If these assumptions and experimental results are taken into consideration, especially if inhibitory inputs to the AV node may be operative and if a slow ventricular response during atrial fibrillation may be induced at least in part by these inhibitory stimuli or processes, a random walk model or a diffusion equation approximation can be thought to be valid. If this diffusion equation can be solved under appropriate conditions, a more detailed knowledge of the function of the AV node and of the action of digitalis on the AV node in atrial fibrillation could be obtained.

\section{Appendix}

1. Some basic stochastic processes :1,2),8),97 The simplest example of stochastic processes is a Poisson process, whose parameter $\lambda$ is a constant with the dimension of the reciprocal of time. It will measure the mean rate of occurrence of events over a long period of time. We consider the number of events (for example, the number of firing of a neuron), $x$, occurring in an arbitrary interval of length $t$. Then $\mathrm{x}$ has a Poisson distribution of mean $\lambda t$ i.e., the probability density function $\operatorname{Px}(t)$ is

$$
P_{x}(t)=\frac{(\lambda t)^{x}}{x !} \exp (-\lambda t)
$$

The distribution of the intervals between events occurring according to a Poisson process is called exponential. The probability density function $I(t)$ of the exponential distribution of parameter $\lambda$ is

$$
\mathrm{I}(\mathrm{t})=\lambda \exp (-\lambda \mathrm{t})
$$

The following model is considered after relaxing the assumptions imposed by Stein :9) (1) excitatory impulses occur randomly with a frequency $\lambda / \mathrm{sec}$; (2) each impulse produces unit depolarization; (3) if the depolarization reaches a threshold of $\mathrm{k}$ units, the neuron fires. If these assumptions would obtain, the exact form of the interresponse time distribution for all $\mathrm{k}$ is the Gamma distribution : If there are $\lambda$ impulses/sec, the probability that there will be an impulse in a short time, $\mathrm{dt}$, is simply $\lambda \mathrm{dt}$. The probability of exactly $\mathrm{k}-1$ impulses in a time interval $\mathrm{t}$ is given by a Poisson distribution with parameters $\lambda, t$, and $\mathrm{k}-1$; namely, $\mathrm{f}(\mathrm{t})$ expressing the probability density function in this case equals to $(\lambda \mathrm{t})^{\mathrm{k}-1} \exp (-\lambda \mathrm{t}) /(\mathrm{k}-\mathrm{l}) !$ according to the equation (1). The probability that the $\mathrm{k}$-th impulse will occur between times $t$ and $t+d t$, namely $f(t) d t$, is the product of these 2 and is expressed by following equation: 


$$
f(t)=\frac{\lambda^{\mathrm{k}} \mathrm{t}^{\mathrm{k}-1}}{(\mathrm{k}-1) !} \exp (-\lambda \mathrm{t})
$$

When $\mathrm{k}$ takes integer values as mentioned above, this distribution is sometimes known as an Erlangian distribution and arises as the sum of $\mathbf{k}$ exponentially distributed random variables.

When $\mathrm{k}$ is not always equal to integer values, this distribution is called a Gamma distribution; the probability density function is

$$
f(t)=\frac{b^{a} t^{a-1}}{\Gamma(a)} \exp (-b t)
$$

where $\Gamma(\mathrm{a})$ is the Gamma function, and $\mathrm{a}$ and $\mathrm{b}$ are arbitrary real positive numbers. Moreover, for $b=1 / 2$ and $a=k / 2$, where $k$ is an integer, $f(t)$ becomes a probability density function of chi-squared distribution with $\mathbf{k}$ degrees of freedom. Therefore the test of goodness-of-fit for an Erlangian distribution can be performed by the use of the table of chi-squared distribution as in the text.

2. Information theory: ${ }^{10)}$ The entropy $\mathrm{H}_{0}(\tau)$ of random interval distributions consisting of discrete variables of time such as $\mathrm{R}-\mathrm{R}$ intervals in atrial fibrillation can be calculated from the interval histogram, and the conditional entropy $\mathrm{H}_{1}(\tau)$ from the transition matrix categorized by the duration of these intervals, in steps of $\tau$ msec. Therefore, the entropy $\mathrm{H}_{0}(\tau)$ is defined by

$$
\mathrm{H}_{0}(\tau)=-\sum_{\mathrm{i}} \mathrm{p}(\mathrm{i} ; \tau) \log _{2} \mathrm{p}(\mathrm{i} ; \tau)
$$

where $p(i ; \tau)$ is the probability of the i-th interval, which is counted by steps of $\tau$ msec. Similarly the conditional entropy $\mathrm{H}_{1}(\tau)$ is defined by

$$
H_{1}(\tau)=-\sum_{i} \sum_{j} p(i, j ; \tau) \log _{2} p_{i}(j ; \tau),
$$

where $p(i, j ; \tau)$ is the joint probability of the $i$-th and the $j$-th interval, and $p_{i}(j ; \tau)$ is the conditional probability of the $\mathrm{j}$-th interval under the condition that the $\mathrm{i}$-th interval occurred just before the $j$-th interval, each interval being counted by steps of $\tau$ msec respectively. Hereafter we neglect the time scale $\tau$ except otherwise stated.

The value of entropy of Gaussian distribution is closely related with the standard deviation $\sigma$ of the distribution. In the case of one-dimensional Gaussian distribution, the probability density function is given by

$$
\mathrm{p}(\mathrm{x})=\frac{1}{\sqrt{2 \pi} \sigma} \exp \left(-\frac{\mathrm{x}^{2}}{2 \sigma^{2}}\right)
$$

Shannon and Weaver ${ }^{4}$ obtained the following equation for $\mathbf{H}$ :

$$
\mathrm{H}_{0}=\frac{\log _{2}(2 \pi \mathrm{e})^{1 / 2} \sigma}{\log _{2} \mathrm{e}}
$$

Similarly in the case of $\mathrm{n}$-dimensional Gaussian distribution, the probability density function is given by

$$
p\left(x_{1}, \ldots, x_{n}\right)=\frac{\left|a_{i j}^{n}\right|^{-1 / 2}}{(2 \pi)^{n / 2}} \exp \left(-\frac{1}{2} \sum a_{n}^{1 j} x_{1} x_{j}\right)
$$


where $\left|\mathbf{a}_{1 j}^{\mathbf{n}}\right|$ is the determinant of the covariance matrix $\left(a_{1 j}^{n}\right)$ whose elements are $a_{i j}^{n}$, and $a_{11}^{1 j}$ is the element of the inverse matrix of the covariance matrix $\left(a_{1 j}^{n}\right)$. The entropy of the n-dimensional Gaussian distribution becomes

$$
\mathrm{Z}_{\mathrm{n}}=\frac{\left.\log _{2}(2 \pi \mathrm{e})^{\mathrm{n} / 2} \mid \mathrm{a}_{1 \mathrm{j}}^{\mathrm{n}}\right\rfloor^{1 / 2}}{\log _{2} \mathrm{e}}
$$

The necessary and sufficient condition for the sequence of R-R intervals in atrial fibrillation to be the $m$-th order Markov process is described by the following relation :

$$
\mathrm{H}_{1}(\tau) \geqq \mathrm{H}_{2}(\tau) \geqq \ldots \geqq \mathrm{H}_{m-1}(\tau)>\mathrm{H}_{m}(\tau)=\mathrm{H}_{m+1}(\tau)=\ldots,
$$

where $H_{i}(\tau)$ is the conditional entropy of the $\mathrm{i}$-th order.

The conditional entropy of the $i$-th order $H_{1}(\tau)$ is equal to the following equation of the joint entropy:

$$
\mathrm{H}_{\mathrm{i}}(\tau)=\mathrm{Z}_{\mathrm{i}+1}(\tau)-\mathrm{Z}_{\mathrm{i}}(\tau)
$$

where $Z_{\mathrm{i}}(\tau)$ is the joint entropy of the $\mathrm{i}$-th order. If we apply Eq. (12) to Eq. (11), the necessary and sufficient condition to be the m-th order Markov process is shown in terms of the joint entropy $Z_{i}(\tau)$.

In the case of n-dimensional normal distribution, $n$-dimensional joint entropy $Z_{n}$ is given by Eq. (10). Therefore the following equations hold for the conditions of the $\mathrm{m}$-th order Markov process which is given by the terms of covariance matrix $\left(a_{i j}^{p}\right)$ :

for all the integers n's less than the integer $m$

$$
\left.\left|a_{i j}^{n-1} \geqq\right| a_{i j}^{n-2}\right|^{1 / 2}\left|a_{i j}^{n}\right|^{1 / 2}
$$

for the integer $n$ equal to the integer $m$

$$
\left|a_{i, j}^{n-1}\right|>\left|a_{i j}^{n-2}\right|^{1 / 2}\left|a_{i j}^{n}\right|^{1 / 2},
$$

and for all the integers n's greater than the integer $m$

$$
\left|a_{1 j}^{n-1}\right|=\left|a_{i j}^{n-2}\right|^{1 / 2}\left|a_{i j}^{n}\right|^{1 / 2} \text {. }
$$

Each element $a_{x y}$ of the covariance matrix $\left(a_{i j}^{n}\right)$, that is, the covariance of the sequence $\mathrm{x}$ and $\mathrm{y}$ is given by

$$
\mathrm{a}_{x y}=\frac{\sum_{\mathrm{i}}\left(\mathrm{x}_{\mathrm{i}}-\mathrm{m}_{x}\right)\left(\mathrm{y}_{\mathrm{i}}-\mathrm{m}_{\bar{\gamma}}\right)}{\mathrm{N}},
$$

where $m_{x}$ and $m_{y}$ are the means of the sequence $x$ and $y$, respectively. When one considers some mappings among the sequences $x, y, z, \ldots$, there exists a mapping from the sequences $x, y, z, \ldots$ to the sequence $x$ : the variable $y_{i}$ is substituted by $x_{i+1}, z_{i}$ by $x_{i+2}, \ldots$ By this mapping, Eq. (16) becomes the following equation:

$$
a_{x_{i}}, x_{i+j}=\sigma_{x}^{2}\left\{\frac{\sum_{i}^{N-j}\left(x_{i}-m_{x}\right)\left(x_{i+1}-m_{y}\right)}{(N-j) \sigma_{x}^{2}}\right\}=\sigma_{x}^{2} \cdot \rho_{i, i+j},
$$


where $\sigma_{\mathrm{x}}$ is the variance of $\mathrm{x}$ and $\rho_{1, \mathrm{i}+\mathrm{j}}$ is the serial correlation coefficient between the variables $x_{i}$ and $x_{i+j}(i=l \sim(N-j))$. From the properties of the covariance matrix of the sequence of $R-R$ intervals it is clear that

$$
a_{x_{i}}, x_{i+j}=a_{x_{i+j}}, x_{i} \text {, and } a_{x_{i}}, x_{i}=\sigma_{x}^{2} \text {. }
$$

We rewrite $\rho_{\mathrm{i}, \mathrm{i}+\mathrm{j}}$ by $\rho_{\mathrm{j}}$ and call $\rho_{\mathrm{j}}$ by the $\mathrm{j}$-th order serial correlation coefficient.

Thus the determinant of the covariance matrix of the sequence of R-R intervals is given by

$$
\left|a_{i j}^{n}\right|=\sigma^{2 n}\left|\begin{array}{ccr}
1 & \rho_{1} & \rho_{2} \ldots \ldots \rho_{n-1} \\
\rho_{1} & 1 & \rho_{1} \ldots \ldots . \rho_{n-2} \\
\cdot & & \vdots \\
\rho_{n-1} & \rho_{n-2} & \ldots \ldots \ldots \ldots .
\end{array}\right| \equiv \sigma^{2 n}\left|S^{n}\right|
$$

where $\left|S^{n}\right|$ denotes the determinant of the serial correlation coefficients. Hence the necessary and sufficient condition to be the m-th order Markov process is given, in terms of the serial correlation coefficients, from the Eqs. (13) to (15):

for all integers $n$ 's less than the integer $m$

$$
\left|S^{n-1}\right| \geqq\left|S^{n-2}\right|^{1 / 2}\left|S^{n}\right|^{1 / 2}
$$

for the integer $n$ equal to the integer $m$

$$
\left|\mathrm{S}^{\mathrm{n}-1}\right|>\left|\mathrm{S}^{\mathrm{n}-2}\right|^{1 / 2}\left|\mathrm{~S}^{\mathrm{n}}\right|^{1 / 2}
$$

and for all the integers $n$ 's greater than the integer $m$

$$
\left|\mathrm{S}^{\mathbf{n}-1}\right|=\left|\mathrm{S}^{\mathrm{n}-2}\right|^{1 / 2}\left|\mathrm{~S}^{\mathrm{n}}\right|^{1 / 2}
$$

We apply Eqs. (19) to (21) to estimate the order of Markov process.

\section{REFERENCES}

1. Cox DR: Renewal Theory. Methuen, London, 1962

2. Cox DR, Lewis PAW: The Statistical Analysis of Series of Events. Methuen, London, 1966

3. Blackman RB, Tukey JW: The Measurement of Power Spectra. Dover, New York, 1958

4. Shannon C, Weaver W: The Mathematical Theory of Communication. Univ of Illinois Press, Urbana, 1949

5. Hashida E, Yoshitani N, Tasaki T: A probabilistic study of the irregularity of R-R interval durations in atrial fibrillation. Jap Circulat J 37: 1423, 1973

6. Wilks SS: Mathematical Statistics. 2nd Ed, Wiley, New York, 1962

7. Gramér H: Mathematical Methods of Statistics. Princeton Univ Press, Princeton, NJ, 1963

8. Feller W: An Introduction to Probability Theory and Its Applications. Vol. II, Wiley, New York, 1966

9. Stein RB: A theoretical analysis of neuronal variability. Biophys J 5: 173, 1965

10. Nakahama H, Ishii N, Yamamoto $M$ : Markov process of maintained impulse activity in central single neurons. Kybernetik 11: 61,1972

11. Perkel DH, Gerstein GL, Moore GP: Neuronal spike trains and stochastic point processes. 
I. The single spike train. Biophys J 7: 391, 1967

12. Khintchine AY: Mathematical Methods in the Theory of Queueing. Griffin, London, 1960

13. Gerstein GL, Mandelbrot B: Random walk models for the spike activity of a single neuron. Biophys J 4: 41, 1964

14. Fienberg SE: A biometrics invited paper-stochastic models for single neuron firing trainsA survey. Biometrics 30: 399, 1974

15. Johannesma PIM: Diffusion models for the stochastic activity of neurons. in Neural Networks, ed by Caianiello ER, Springer, New York, p116-144, 1968

16. Moore $\mathbf{E N}$ : Observations on concealed conduction in atrial fibrillation. Circulat Res 21 : 201,1967

17. Moe GK, Abildskov JA: Observations on the ventricular dysrhythmia associated with atrial fibrillation in the dog heart. Circulat Res 14: 447, 1964

18. Langendorf $\mathrm{R}$, Pick A, Katz LN: Ventricular response in atrial fibrillation: Role of concealed conduction in the A-V junction. Circulation 32: 69, 1965

19. Stein RB: The stochastic properties of spike trains recorded from nerve cells. in Stochastic Point Processes, ed by Lewis PAW, Wiley, New York, p 700-731, 1972

20. Ten Hoopen M, Reuver HA: Selective interaction of two independent recurrent processes. J Appl Prob 2: 286, 1965

21. Bishop PO, Levick WR, Williams WO: Statistical analysis of the dark discharge of lateral geniculate neurons. J Physiol 170: 598, 1964

22. Levick WR, Williams WO: Maintained activity of lateral geniculate units in darkness. J Physiol 170: 582, 1964

23. Mendez G, Moe GK: Some characteristics of transmembrane potentials of AV nodal cells during propagation of premature beats. Circulat Res 19: 993, 1966

24. Iso Y: Threshold elements and random process. I. The first passage problem for Erlang's system. Rep Kyushu Inst Tech 15: 13, 1965

25. Zipes DP, Mendez C, Moe GK: Evidence for summation and voltage dependency in rabbit atrioventricular nodal fibers. Circulat Res 32: 170, 1973 